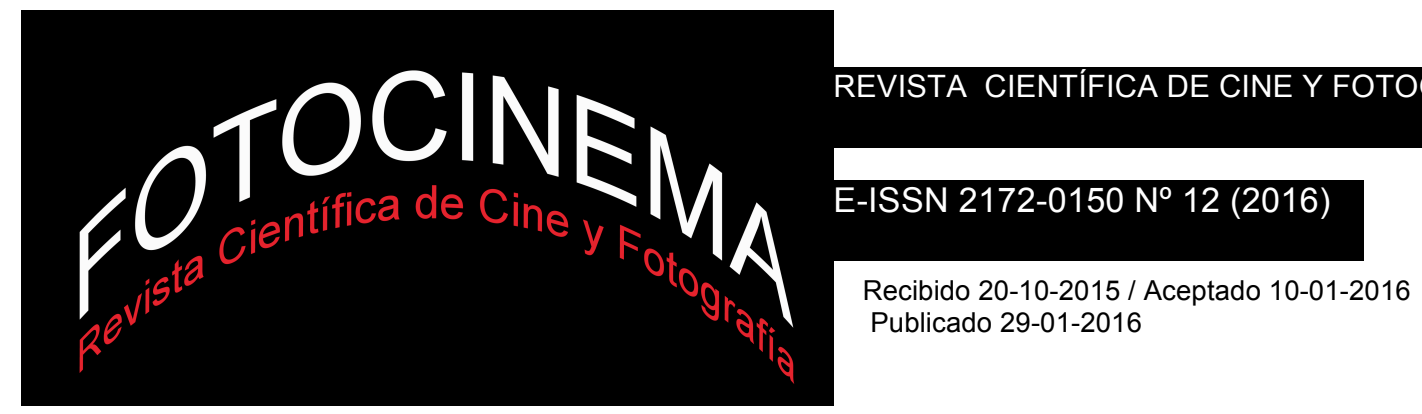

\title{
IDENTIDADES ESTIGMATIZADAS Y SU REFIGURACIÓN PERFORMATIVA EN CÉSAR DEBE MORIR
}

\section{STIGMATIZED IDENTITIES AND THEIR PERFORMATIVE REFIGURATION IN CAESAR MUST DIE}

\author{
Moira Pérez \\ Universidad de Buenos Aires/Universidad Nacional de Lomas de Zamora, Argentina \\ perez.moira@gmail.com
}

Gilda Bevilacqua

Universidad de Buenos Aires, Argentina gildasbevilacqua@gmail.com

\section{Resumen:}

El filósofo Ian Hacking ha afirmado, siguiendo a Sartre: "Posibilidad, proyecto y prisión son una misma cosa". En ese trabajo, nos interesa seguir esta pista utilizando como desencadenante del análisis el film César debe morir (Paolo y Vittorio Taviani, 2012), un docuficción que relata la experiencia de un grupo de prisioneros que ensayan y ponen en escena la obra Julio César de Shakespeare, entrelazando ese hilo con la vida cotidiana de los reclusos y con la trama de la obra misma. Mediante el film, sus recursos, y lo que muestra de la experiencia teatral, reflexionaremos sobre los vínculos entre posibilidad, proyecto y prisión - literal, y metafórica. Nuestro objetivo es analizar la relación entre la construcción de identidades estigmatizantes y las posibilidades de desplazamiento en términos de agencia y empoderamiento. Consideraremos, además, los modos en que el teatro y el cine pueden resultar medios privilegiados para llevar adelante la reconfiguración performativa de aquellas descripciones. Para ello, tomaremos como clave de lectura una serie de nociones provenientes de la filosofía contemporánea, principalmente aquella de "clases interactivas", aportada por el mismo Hacking, la de "performatividad", siguiendo el análisis de Judith Butler, y la de "matriz", que es utilizada por ambos autores.

\begin{abstract}
:
Philosopher Ian Hacking has asserted, following Sartre: "Possibility, project and prison are one of a piece". In this work, we are interested in following that cue through an analysis of the film Caesar must die (Paolo and Vittorio Taviani, 2012), a docufiction portraying the experience of a group of prisoners who rehearse and stage William Shakespeare's Julius Caesar. The film blends this with the prisoners' everyday life and with the plot of the classical play. Through the film, its resources, and what it shows about the theatrical experience, we will address the relationship between possibility, project, and prison - literal, and metaphoric. Our aim is to analyze the relationship between the construction of stigmatized identities and the possibilities of displacement in terms of agency and empowerment. We will also consider the ways in which theatre and cinema could be particularly useful means to carry out this performative reconfiguration of those descriptions. In order to achieve this, we will adopt as a key a number of notions from contemporary philosophy, notably "interactive kind", brought by Hacking himself, "performativity", following Judith Butler's analysis, and the idea of "matrix", adopted by both authors.
\end{abstract}

\section{Palabras clave:}

Matriz; performatividad; prisión; clase interactiva; arte.

Keywords:

Matrix; performativity; prison; interactive kind; art. 


\section{Introducción}

El filósofo canadiense Ian Hacking ha afirmado, siguiendo a Jean-Paul Sartre: "Posibilidad, proyecto y prisión son una misma cosa" (Hacking, 2000, p. 109). Con estas breves palabras el autor abre una ventana a la enorme complejidad contenida en los procesos sociales y de subjetivación, superando el simplismo tanto del voluntarismo como del determinismo. Es esa complejidad la que encontramos en los procesos que analizamos en este trabajo, donde seguimos la sugerencia de Hacking para indagar no sólo en lo que contienen los vínculos entre prisión, posibilidad y proyecto, sino también -y sobre todo- en sus interacciones con el arte y las posibilidades que habilita.

El desencadenante del análisis será un film en el que posibilidad, proyecto, prisión y arte se encuentran de modo explícito y singular. Se trata de César debe morir (Cesare deve morire, Paolo y Vittorio Taviani, 2012), un docuficción que relata la experiencia de un grupo de prisioneros que ensayan y ponen en escena la obra Julio César de William Shakespeare, entrelazando ese hilo con la vida cotidiana de los reclusos y con la trama de la obra misma. Tal como veremos, esta película habilita numerosas líneas de análisis interesantes, de las cuales aquí tomaremos sólo algunas. Principalmente, nos interesa repensar con el film, y en la clave del marco teórico elegido, el vínculo entre la construcción de identidades en un contexto restrictivo, y las posibilidades de desplazamiento en términos de agencia y empoderamiento. Veremos, además, que el teatro y el cine (hacia adentro y hacia afuera del entorno punitivo) se muestran como medios privilegiados para llevar adelante la reconfiguración performativa de las identidades. Tienen el potencial de colaborar para modificar la auto-percepción de los sujetos ${ }^{1}, y$ facilitar un efecto "suspensor" que permite la distancia necesaria para improvisar un desplazamiento respecto de los mandatos de la matriz y la historia que los sustenta.

\footnotetext{
${ }^{1}$ En este artículo hemos procurado reducir los usos de sustantivos universales generizados, pero en los casos en que sí se mencionan (tal como esta alusión a "los sujetos"), utilizaremos el género masculino a modo de universal. En lo que hace a los presos de Rebbibbia, aclaramos desde ya que el film retrata un grupo que se identifica en su totalidad con ese género.
} 
Para llevar adelante este análisis utilizaremos un marco teórico proviene de la filosofía contemporánea, y en particular del trabajo de Hacking mismo y de la filósofa norteamericana Judith Butler. Trabajaremos con las nociones de "clases interactivas" y "efecto bucle" aportadas por el canadiense, la de “performatividad” que desarrollara Butler, y la de "matriz", utilizada por ambos autores. A lo largo de las últimas décadas, estas nociones han sido de gran utilidad para indagar en la (re)construcción de identidades abyectas del pasado y del presente, sus limitaciones y posibilidades. Aquí, las adoptaremos con dos objetivos centrales: por un lado, comprender los procesos de construcción de ciertas identidades en el contexto de una matriz restrictiva; por el otro, indagar en las posibilidades de rearticulación o refiguración que habilita su comprensión en tanto clases interactivas y/o performativas, particularmente a través de la intervención del arte.

Nuestra metodología combina el análisis teórico de las categorías elegidas, a partir de la reconstrucción y desarrollo de los conceptos, con su aplicación al caso de César debe morir. Este segundo aspecto resulta particularmente importante teniendo en cuenta que nuestra tesis introduce un elemento nuevo a la tríada con la que comenzamos el trabajo: a la posibilidad, el proyecto y la prisión se suma el arte, que entendemos como un medio para realizar una transformación en el horizonte cotidiano de los protagonistas del film.

El trabajo se estructura de acuerdo a dos ejes conceptuales centrales, que responden a las categorías citadas: el de la matriz en la que se insertan las descripciones, por un lado, y el de la performatividad y el desplazamiento de las clases interactivas, por el otro. Estos ejes, a su vez, pueden ser ubicados en distintos momentos del proceso de desarrollo de los protagonistas del film: las condiciones en que se encuentran al encarar el proyecto artístico (momento en el que serán particularmente útiles las nociones de "matriz" y de “acciones bajo descripciones”), y qué procesos y posibilidades se abren a partir de la interacción de los protagonistas con el arte (para lo cual nos servirá remitirnos al concepto de "performatividad" y profundizar en las “clases interactivas”). 
Cada uno de esos dos ejes conceptuales ocuparán las secciones tercera y cuarta del trabajo. Antes de sumergirnos en ellas, expondremos algunos puntos fundamentales sobre el film, incluyendo su trama, sus recursos, y algunas líneas de análisis a las que puede dar lugar. Posteriormente, en el tercer apartado pasaremos a indagar en los mecanismos de estigmatización y delimitación de las posibilidades del sujeto, utilizando la noción de "matriz" de Hacking. En tercer lugar, veremos de qué manera puede darse una transformación de esas descripciones estigmatizantes, y para ello recurriremos al análisis butleriano de la "performatividad" y a las ideas de Hacking de "clase interactiva" y "efecto bucle". En ambos apartados proponemos una retroalimentación entre las categorías y la obra de los Taviani: los conceptos nos permitirán comprender mejor los contenidos y operaciones del film, y este último servirá de herramienta para una comprensión más profunda de lo que implican aquellas categorías. Esto es particularmente relevante en el análisis de la performatividad, ya que César debe morir nos permite abordar las posibilidades que puede habilitar el arte - en este caso, teatro y cine - para efectuar aquella transformación performativa de los sujetos. Finalmente, un quinto apartado nos servirá para ofrecer algunas reflexiones a modo de conclusión.

\section{César debe morir}

César debe morir ${ }^{2}$ es un film difícil de clasificar netamente en un género; es posible afirmar que esa ambigüedad está relacionada justamente con las posibilidades tanto estéticas como analíticas que nos brinda y abre en tanto ejercicio de arte. Dirigido por los hermanos Paolo y Vittorio Taviani y estrenado en el año 2012, este “docuficción” entrelaza el género documental con la ficción para relatar la experiencia de un grupo de personas privadas de

${ }^{2}$ Ficha técnica / Título original: Cesare deve morire / Año: 2012 / Duración: 76 min. / País: Italia / Directores: Paolo Taviani, Vittorio Taviani / Guión: Paolo Taviani, Vittorio Taviani (Historia: William Shakespeare) / Música: Giuliano Taviani, Carmelo Travia / Fotografía: Simone Zampagni / Reparto: Fabio Cavalli, Salvatore Striano, Giovanni Arcuri, Antonio Frasca, Juan Dario Bonetti, Vincenzo Gallo, Rosario Majorana, Francesco De Masi, Gennaro Solito, Vittorio Parrella, Pasquale Crapetti, Francesco Carusone, Fabio Rizzuto, Fabio Cavalli, Maurilio Giaffreda/ Productoras: Kaos Cinematografica, Rai Cinema, Stemal Entertainment, Le Talee / Género: Drama / Idioma: italiano. 
su libertad, calificadas como "de alta peligrosidad", en la cárcel romana de máxima seguridad de Rebibbia. Este grupo de hombres participa de los talleres teatrales organizados por el director Fabio Cavalli, mientras cumplen su condena a penas severas (de 15 años a reclusiones perpetuas). En esta ocasión, el grupo trabajó con la obra Julio César de William Shakespeare, seleccionada especialmente por los directores Taviani para ser puesta en escena y rodada.

El film está estructurado en tres grandes secuencias, delimitadas y vinculadas por dos recursos estético-narrativos entrelazados de modo evidente: el flashback y la fotografía. El primer recurso une el comienzo del film (primera secuencia) con el final (tercera y última), momento en el que se retoma (y repite en parte) la secuencia inicial. En ambas vemos la puesta en escena "real" y el estreno público de la obra, y posteriormente la vuelta de los reclusos a sus celdas. En un momento intermedio, la segunda secuencia (la más extensa) muestra la presentación frente a los reclusos del proyecto de teatro y la obra elegida, el casting y el desarrollo del ensayo de la obra en el contexto de la cárcel y de su situación de encierro. La fotografía acompaña esta temporalidad diferida planteada por el flashback, mediante el paso del color de la secuencia inicial al blanco y negro (casi gris) de la segunda, y el retorno al color en la tercera.

La primera secuencia muestra el estreno de la obra, y comienza con la escena en la que Brutto, el personaje principal, pide a sus compañeros que lo ayuden a morir. Brutto cae muerto. Termina la representación de la obra. El público aplaude de pie. La sala de teatro ahora está vacía. Los actores se van del escenario. Cambio de escena. Los hombres entran a la cárcel. Caminan por sus pasillos. Entra cada uno a su celda. Fin de la secuencia. En una escena ya en blanco y negro, un texto sobreimpreso dice: "Seis meses antes". Así comienza la segunda secuencia del film, que muestra de manera cronológica escenas representativas de los seis meses de preparativos y ensayos previos al estreno de la obra, alternados con la vida cotidiana de los reclusos. Es difícil delimitar si lo que se está representando es el ensayo en el contexto de la vida cotidiana y de las instalaciones presidiarias, o si éstas han cambiado, al 
menos en el tiempo de rodaje del film, para devenir ensayo y escenario. La tercera y última secuencia retoma el color y una escena específica de la obra, que es anterior a la que dio inicio a la primera: el enfrentamiento entre los ejércitos de Octavio y Brutto. Se muestran fragmentos de las escenas siguientes, repitiendo de este modo el tramo inicial del film, el final de la obra, los aplausos del público de pie. En esta última secuencia se añade a eso el festejo subsiguiente, la alegría, los saludos y aplausos de los actores. El cambio de escena nos lleva directo a las puertas de las celdas de los personajes protagonistas (del film y de la obra) y su ingreso en ellas: César, Brutto, Casio. Este último, ya en el interior de su celda y su intimidad, mientras prepara sus cosas para hacerse un café, mira a cámara y afirma: "Desde que conocí el arte, esta celda se ha convertido en una prisión”. Corte a negro. Se comienza a proyectar, en blanco y negro, a pantalla partida, un recuadro con las imágenes de cada interno en el casting de la obra, y un texto sobreimpreso que relata brevemente cómo siguió la vida de algunos de ellos.

La primera y última secuencias nos muestran así dos caras de un mismo espacio concreto, dos situaciones que coexisten: la prisión como lugar de encierro, de la repetición de lo mismo, y la prisión como lugar de apertura, de posibilidad de surgimiento de algo “otro". Esta "realidad” bifronte está enfatizada mediante la fotografía, con el color de sus escenas. Para los directores del film,

el blanco y el negro es irreal, mientras que, por el contrario, el color es naturalista y realista. En el pasado, el cine era en blanco y negro. Entonces, utilizar el blanco y el negro es hacerle una violencia a la realidad, pero la realidad no es en blanco y negro. La realidad es a color. Es una violencia que nosotros hicimos así como es de violenta la película que hemos contado (Suraci, 2013).

Entendemos que esta "violencia" es el modo de representar una violencia que está en el ambiente retratado en el film. En la prisión, aun existiendo algunos "planos a color" (la realidad como un instante vivible, como un acontecer diferente), el ambiente "gris" es abrumadoramente predominante durante la mayor cantidad de la vida de sus protagonistas (como calendario y como 
horas reloj) - y, por consiguiente, durante la mayoría del tiempo del film. Esa es la violencia del encierro, y parece ser también la violencia de los motivos del encierro, ya que en el film ninguno de los protagonistas-actorespersonajes cuestiona la privación de su libertad. Todo esto pone de relieve la potencia de los recursos cinematográficos utilizados, en relación con los múltiples significados que pueden tener tanto la idea de encierro como la de libertad (palabra que, por otra parte, aparece repetidas veces en el transcurso de los ensayos).

En esta película, como decíamos al principio, convergen distintos planos de representación y aspectos de análisis posibles, que la hacen una obra rica y polifacética. Teniendo en cuenta el cruce que propusimos al inicio entre el film y las nociones teóricas señaladas, podemos destacar particularmente algunos de dichos aspectos:

1) La tensión y el entrecruzamiento entre el plano de lo "real" (la representación de la cotidianidad en la cárcel) y lo "ficticio" (la representación de los ensayos de la obra, en el marco de esa cotidianidad, y de su estreno ante el público).

2) La tensión entre la identidad de "ser un preso/estar preso", y la dimensión artística que estas personas en situación de encierro incorporan a sus vidas cotidianas.

3) El vínculo entre arte y vida, expresado en dos planos. Por un lado, vemos la práctica artística teatral como medio para que los internos re-tramen sus vidas y su pasado, y los transiten de otro modo. Por el otro, a través del film que protagonizan aparece el cine como modo de hacer pública esa nueva trama, ese cambio íntimo y personal, gracias a su "reproductibilidad técnica”, en términos benjaminianos.

4) El uso y transitar de los espacios carcelarios, su transformación y la transformación performativa de las identidades, tanto de los objetos como de los sujetos "encerrados". El espacio de la cárcel se transfigura en la escenografía móvil de los ensayos. Pero no sólo se transforma el espacio, sino también las personas que lo habitan de ese nuevo modo. 
5) La obra de teatro elegida en sí misma, y la versión que realiza el grupo de teatro de los internos; los vínculos metafóricos entre la "realidad" de los presos y la "realidad" de cada personaje y del conjunto, ya que no es cualquier obra, y su trama trágica genera identificación y empatía en los reclusos.

6) El cine como práctica colectiva e interactiva que modifica a cada una de las partes que participan en ella: realizadores, protagonistas-actores, entrevistados, extras, espectadores, entre otros.

Cada uno de estos aspectos son inescindibles en la práctica, y se alimentan mutuamente. Por otro lado, hecha esta distinción analítica en seis líneas posibles de indagación vemos que cada una de ellas, por sus características e implicancias, requeriría un análisis particular y profundo. En nuestro trabajo abordaremos como objeto de estudio los primeros cuatro ejes, ya que es en ellos donde se ponen en juego de manera más patente las categorías con las que nos proponemos trabajar. Consideraremos a los cuatro ejes de manera interrelacionada, teniendo en cuenta también que el quinto y sexto pueden ser entendidos en cierta forma como variables o desprendimientos más exhaustivos de los precedentes. Esperamos que nuestra propuesta sirva de invitación a continuar el análisis, profundizarlo, y aportar perspectivas acerca de lo que aquí hemos tenido que dejar al margen.

\section{La matriz: límites y posibilidades}

Tal como adelantamos en nuestra introducción, el primer objetivo de este trabajo es comprender los modos en los que la matriz orienta las construcciones identitarias que se dan dentro de ella. Esto se vincula a su vez con nuestra segunda finalidad: para pensar en el potencial transformador de las representaciones artísticas, tales como el cine y el teatro, es necesario atender, ante todo, al panorama en el que éstas se insertan. Básicamente: ¿qué es eso que las representaciones podrían (ayudar a) transformar? En esta sección del trabajo buscaremos responder a dicho interrogante, entendiendo 
que la estigmatización 3 instala un escenario de restricciones en el que luego (en el siguiente apartado) se insertarán los cambios habilitados por una representación performativa. Concretamente, si "posibilidad, proyecto y prisión son una misma cosa”, ahora quisiéramos detenernos específicamente en lo que hace a la "prisión", entendida en sentido tanto literal como metafórico. Antes de adentrarnos en este análisis, sin embargo, es importante aclarar que al referirnos a "posibilidad" y "prisión" no estamos aludiendo a una diferenciación neta o tajante. No existe, en realidad, un “antes" (la prisión) y un "después” (la posibilidad), sino más bien la interacción de distintas identidades y actuaciones que se superponen, se (re)producen y se contradicen.

Uno de los planos de análisis de la película César debe morir que mencionamos en el apartado anterior es el de la cotidianidad de los internos en Rebbibbia. Este aspecto, que nos servirá para indagar en el proceso de construcción identitaria, emerge de manera intermitente a lo largo de la película. Por ejemplo, en una escena en la que se muestra a un preso ensayando, luego ese plano se amplía hasta que entra en cuadro su compañero de celda, que expresa escepticismo o resignación ante la cotidianidad de la vida en la prisión. Esta escena, como muchas otras, nos devuelve a la crudeza de la prisión como matriz institucional, tal como la entiende Ian Hacking. Veamos en detalle esta idea de matriz y su relación con los sujetos, para luego analizar, en la siguiente sección, los modos posibles de incidencia en ella.

¿Qué es exactamente esta matriz? ¿Cómo nos condiciona? El autor utiliza el término para referirse a un contexto social y material, una serie de instituciones, lugares físicos, prácticas y reglamentaciones que nos atraviesan. Necesita este concepto porque, en su teoría, "las ideas no existen en el vacío. Habitan un contexto social”, lo cual lo lleva a proponernos que llamemos a dicho contexto "la matriz en la que se forma una idea, un

\footnotetext{
3 En nuestro uso de los términos "estigma" y "estigmatización" seguimos a Goffman, quien entiende estigma como "la situación del individuo inhabilitado para una plena aceptación social" (Goffman, 2006, p. 7), debido a la relación que existe entre sus atributos y los estereotipos o expectativas asignados al grupo social al que pertenece.
} 
concepto o clase" (Hacking, 1999, p. 10). A Hacking le interesa pensar no en los individuos, o cada persona particular, sino más bien en las clasificaciones, las ideas con las que son catalogadas o descriptas dichas personas. Y estas clasificaciones e ideas se dan en el marco de lo que él denomina "matriz". Claro que esas matrices influyen sobre la subjetividad: en palabras de Butler, quien utiliza el mismo término para considerar específicamente la configuración del género, "el sujeto es producido dentro de una matriz -y como una matriz- generizada de relaciones" (Butler, 2002, p. 25). Introducir la noción de género en el análisis puede servir para observar que no sólo la matriz es relacional, sino que todas las personas están atravesadas por diversas matrices (siendo la del género sólo una de ellas). Sin embargo, en el caso de César debe morir notamos que existe una que es más fuerte, al menos en principio: aquella del sistema judicial-penitenciario. Nuestros personajes se encuentran en lo que Erving Goffman (2001) ha denominado una "institución total": una matriz que incide en cada una de las esferas de la vida de los internos desde un mismo polo de autoridad 4 .

Es importante no olvidar, a fuerza de centrar la atención sobre su carácter "social”, que la matriz es también material, y en su materialidad afecta profundamente a los individuos que son atravesados por ella. El film nos permite ver cómo esta materialidad se encarna, por ejemplo, en las características espaciales del centro Rebbibbia: un plano tomado desde abajo y contrapicado de uno de los internos ensayando su parte muestra que incluso en el patio su espacio está delimitado; por encima de él, un "techo" de enrejado lo separa del cielo. Lo vemos también en el protocolo de entrada y salida de los internos de sus celdas, al observar cómo, uno tras otro, se paran al lado de su celda, esperan a que se abran ambas puertas, entran, se cierran ambas puertas, y se cierra el pasaplatos. La repetición que ofrece la película de este ritual es una muestra mínima, pero sugerente, de la rutina propia de

\footnotetext{
4 "Una institución total puede definirse como un lugar de residencia y trabajo, donde un gran número de individuos en igual situación, aislados de la sociedad por un período apreciable de tiempo, comparten en su encierro una rutina diaria, administrada formalmente. Las cárceles sirven como ejemplo notorio, pero ha de advertirse que el mismo carácter intrínseco de prisión tienen otras instituciones [sic]" (Goffman, 2001, p. 13).
} 
quien lo vive y vivirá dos o más veces por día, durante todo el resto de su vida.

Ahora bien, retomando el análisis de Hacking, cabe preguntarse: ¿cómo afecta la matriz (las instituciones, las clasificaciones, la materialidad) a las personas que agrupa? ¿cómo interactúan ideas (clasificaciones, etcétera) y objetos (incluidas esas personas)? En un sentido primero y fundamental, las ideas afectan a los objetos en tanto las acciones humanas intencionales son acciones bajo descripciones. Esto significa, ante todo, que a diferente descripción corresponderá diferente comportamiento. Si una persona es clasificada bajo una determinada matriz, sus acciones serán leídas de acuerdo a dicha descripción (Hacking, 1999, p. 31). En nuestro ejemplo, el film nos muestra "prisioneros"; pero esas mismas personas, antes de ingresar en el sistema judicial-penal, no eran "prisioneros" sino "ciudadanos libres", lo cual daba lugar a otro tipo de lecturas (condicionadas, a su vez, por el tipo de descripción que le correspondía en el contexto en el que se movía, y por el cual pudo haber terminado en las manos del sistema penitenciario: "pobre”, "sureño", "peligroso", "inmigrante”, "extranjero", son algunas de las que se ponen en juego en César debe morir).

Por otra parte, también es cierto que podemos actuar solamente bajo las descripciones que tenemos disponibles. $5 \mathrm{Si}$ el sujeto se genera dentro de una matriz, ante todo debemos preguntarnos: ¿qué sujetos se "generan" en esta prisión? El "prisionero" que retratan los hermanos Taviani no puede subsumirse a cualquier tipo de descripción, dado que las mismas condiciones materiales de su matriz lo excluyen de determinados sitios, pertenencias y discursos. El espacio y las rutinas repetidas a lo largo de César debe morir pueden servir como metáfora de esta limitación propia de la matriz, que se hace mucho más evidente en el caso de la prisión, donde las descripciones disponibles son muy limitadas. Al tratarse de una institución total, todos los

5 Cuando abordemos la noción de "performatividad" en el próximo apartado, volveremos sobre la importancia de evitar las interpretaciones ingenuas de este concepto. Ya en Butler aparece tempranamente la advertencia de que la performatividad es "una práctica de improvisación dentro de un escenario constrictivo", ya que "[c]onsiderar al género como una forma de hacer, una actividad incesante performada, en parte, sin saberlo y sin la propia voluntad, no implica que sea una actividad automática o mecánica.” (Butler, 2004, p. 1; subrayado nuestro). 
aspectos de la vida del sujeto están supeditados a la regulación institucional, incluida la posibilidad de la práctica teatral y del rodaje del film que utiliza la prisión como escenario. Sin embargo, como veremos en la sección siguiente, existen vías alternativas para "salir" (entre comillas) de esta restricción... no por azar, creemos, las primeras palabras que escuchamos en la película son “iHuye lejos, Brutto! Huye".

También sabemos que las clasificaciones, a través de sus expresiones tanto simbólicas como materiales, modifican las maneras en las que los individuos se comprenden a sí mismos: son determinantes en nuestras experiencias de agencia, acción, conciencia y auto-conciencia. Esto se da, por supuesto, tanto en el nivel individual como en el colectivo; en muchos casos, la matriz moldea la identidad de un colectivo y, a través de él, la de los individuos que lo componen. La incidencia de la matriz sobre la auto-percepción de los sujetos y sobre su relación con la agencia es fundamental para entender las posibilidades tanto de opresión como de empoderamiento a las que puede dar lugar una clasificación.

Todo esto nos lleva a lo que Hacking denomina "clases interactivas", es decir, aquellas ideas o clasificaciones en las que se da una interacción consciente entre la clase o tipo y la persona que es clasificada. La característica que define a las clases interactivas es que

una vez que son conocidas por las personas [a las que se aplican] o por quienes les rodean, y una vez que comienzan a funcionar en instituciones, [las clases interactivas] modifican las maneras en que los individuos se consideran a sí mismos - e incluso pueden llevar a la gente a modificar sus sentimientos y su comportamiento en parte debido a que reciben esa clasificación. (Hacking, 1999, p. 104) $)^{6}$.

En líneas generales, podríamos decir que, según Hacking, las ciencias sociales trabajan con "clases interactivas", mientras que las ciencias naturales

\footnotetext{
${ }^{6}$ Hacking introduce este concepto a través del ejemplo de la "mujer refugiada" (entendida como clase o tipo, no como una mujer individual específica): "[p]odemos decir que la 'mujer refugiada' (en tanto tipo de clasificación) es una clase interactiva porque interactúa con entidades de esa clase, es decir, personas, incluyendo mujeres refugiadas individuales, que pueden tomar conciencia de cómo son clasificadas y modificar su comportamiento en consecuencia" (Hacking, 1999, p. 32).
} 
lidian con "clases indiferentes" (Hacking, 1999, p. 32). Llevado a nuestro caso, diríamos que las personas privadas de su libertad "son conscientes de lo que se dice de ellas, se piensa de ellas, se hace a ellas" (Hacking, 1999, p. 31), y son afectadas por esto (el docuficción nos muestra cómo deben transitar una serie de espacios, regular su tiempo y rutina diaria, etcétera), mientras que el plutonio no modifica en nada su comportamiento por el sólo hecho de ser clasificado de esa manera.7 Esta idea de clases interactivas es particularmente importante en el caso que estamos analizando, si consideramos lo que hace la experiencia del taller de teatro no sólo sobre los reclusos, sino también sobre, si se quiere, "la idea" de recluso, y la percepción de quien asiste al film.

Es indudable que las matrices condicionan a los objetos y personas que forman parte de ellas, y los determinan con su imponente materialidad. Es cierto que las clasificaciones no sólo afectan, sino que pesan sobre las personas, en muchos casos llevando a situaciones de opresión, violencia e incluso auto-exclusión. Una materialidad que les recuerda permanentemente su condición de marginales, puede terminar logrando que incorporen ese mismo discurso como propio y natural (esto es, naturalizado). Si todo esto es cierto, entonces ¿es la matriz una prisión? César debe morir nos invita a repensar la idea de prisión: la prisión "material", la prisión como figura retórica (como metáfora del encierro y la soledad, o como sinécdoque en tanto institución que representa el sistema opresivo todo), y la prisión como posibilidad. Sin la instancia de prisión como posibilidad, es decir, si esta matriz fuera sólo una prisión, entonces el determinismo sería absoluto y ya no tendríamos ninguna esperanza... Sin embargo, en César debe morir vemos que la misma red que sirve de prisión es fuente de posibilidades.

\footnotetext{
7 Existen, por supuesto, quienes afirman que las "clases indiferentes" no existen ni siquiera en las ciencias llamadas "naturales" o "duras". Lamentablemente, se trata de un debate que excede las dimensiones del presente trabajo.
} 


\section{La performatividad: posibilidades y límites}

Vimos hasta aquí los modos en que nuestras identidades están atravesadas por múltiples matrices, descripciones y clasificaciones, que nos determinan y condicionan tanto en nuestra relación con el entorno (social y material) como en nuestra auto-percepción. Una vez que entendemos que nos encontramos en esta red simbólica y material, ¿qué recursos nos quedan para cumplir con aquel primer pedido que escuchamos en César debe morir, "Huye lejos"? Somos, después de todo, sujetos “construidos" dentro de una matriz: hay ciertas cosas de las que no podemos huir. Sin embargo, precisamente porque somos sujetos "construidos", bien podríamos "construirnos" de otras maneras. Si es cierto que los seres humanos actuamos bajo descripciones, también lo será que "cuando surgen nuevos modos de descripción, surgen como consecuencia nuevas posibilidades de acción” (Hacking, 2000, p. 108). Las descripciones "novedosas" -aún cuando tienen siempre y necesariamente como plataforma de despegue aquellas existentes- pueden dar lugar a nuevas configuraciones materiales, y eventualmente a nuevas matrices, más inclusivas y menos violentas.

¿Cómo se da esa transformación, y qué llegada tiene? Hacking sugiere que la matriz moldea la identidad y auto-percepción de una comunidad y, a través de ella, la de los individuos que la constituyen. A su vez, al tratarse de una clase interactiva, los sujetos mismos influyen sobre la matriz y la modifican, dando lugar a lo que Hacking denomina "looping effect" o "efecto bucle": el fenómeno por el cual la descripción influye en las acciones, y éstas a su vez en la descripción. Hacking es muy explícito al describir las posibilidades de agencia de los sujetos, aun "bajo descripciones":

estos 'tipos de personas' [los que constituyen "clases interactivas”] pueden volverse conscientes de que están siendo clasificados como tales. Pueden hacer elecciones tácitas o incluso explícitas, adaptarse o adoptar maneras de vivir para amoldarse o para evitar las mismas clasificaciones que se les aplican (Hacking, 1999, p. 34).

Como resultado de este proceso, "lo que antes se sabía de un determinado tipo de personas puede tornarse falso, porque las personas de ese tipo han 
cambiado en virtud de lo que piensan de sí mismas" (Hacking, 1999, p. 34). A partir de este fenómeno, la clasificación se modifica y el círculo (el "bucle") comienza nuevamente. César debe morir ofrece un claro ejemplo en esta dirección, sintetizado en su última escena, en boca de Casio: "desde que conocí el arte, esta celda se ha convertido en una prisión”. La celda, convertida forzosamente en un lugar cotidiano de existencia, se retransforma en "prisión” una vez que el recluso mismo se modifica a partir de su contacto con el arte. El "efecto bucle" hace difícil individuar qué influye en qué, o cuál es el inicio de ese espiral: prisión, celda, recluso, arte y vida se afectan mutuamente y ya nada es lo mismo tras el paso de esos seis meses de trabajo artístico.

Este "efecto bucle" es similar al movimiento que, en Butler, sirve de clave para la intervención transformadora. Ese mismo carácter dinámico que el filósofo encuentra en la matriz (que permite que clases, sujetos y acciones se modifiquen entre sí) es el dinamismo que sugiere a Butler la posibilidad de tramar a contrapelo de la norma. Veamos cómo funciona esto, antes de pasar específicamente a ver cómo se ponen en juego estos mecanismos a partir de la intervención del arte.

En concepto de performatividad, proveniente de la filosofía del lenguaje, remite a los actos de habla (o el aspecto de ellos) que producen un evento en el mundo, en lugar de sólo describirlo o relatarlo. Es decir, en los actos de habla performativos el mundo se adapta al discurso, y no viceversa (como sí sucede con los performativos) (Austin, 1975, p. 47). Butler lo toma, junto con distintas observaciones y ampliaciones hechas por filósofos que la precedieron, para aplicarlo al análisis de la construcción del género. Tal como la entiende la autora, la performatividad del género es en gran parte una actuación impuesta, no voluntaria, mediante la cual se negocia la relación con una/s norma/s que es obligatorio citar para ser considerado un sujeto viable (esto es: un ser humano reconocible y comprensible). Sin embargo, la performatividad no existe solamente por la negativa (la construcción de un estigma sobre, en este caso, la persona detenida; las restricciones y violencias que dicha construcción conlleva), sino que también 
tiene una existencia positiva, en tanto posibilidad de (re)definirse performativamente de otra manera. Esto es posible porque, si bien las normas sociales que nos atraviesan logran alterar de manera vital nuestra existencia, su debilidad reside justamente en el hecho de que nunca son normas fijas o definitivas, y su repetición no es ni puede ser una mera réplica de lo mismo. De hecho, Butler ha definido performatividad como "citar la ley para producirla de manera diferente” (Butler, 2002, p. 38). Esta "imperfección" (entre comillas, claro) de la copia deja la ventana abierta para que la norma siempre pueda ser intervenida y alterada a través de una repetición diferente, una performatividad alternativa. En los márgenes de aquella repetición (en ese punto en el que la norma es dinámica y nunca se establece de manera fija y definitiva), reside la posibilidad de rearticular radicalmente -aunque nunca por completo- su horizonte simbólico y las relaciones de poder que conlleva (su matriz), de manera de tornarlos más favorables para la propia vida.

Llegado este punto, cabe incorporar dos aclaraciones adicionales. En primer lugar, algo que adelantamos en el apartado anterior. La performatividad tal como la entiende Butler nunca será "pura”: siempre deberá servirse de los recursos existentes y disponibles en cada caso. En Hacking, vimos esto con las descripciones que tenemos a disposición: no podemos ir más allá de ellas a la hora de pensarnos, tramarnos y ser pensados por otros. En Butler, la performatividad es, como aclaramos desde el inicio, una práctica de improvisación en un escenario de restricciones: no somos totalmente libres, ni estamos totalmente determinados. "Mi agencia no consiste en negar esta condición de mi constitución. Si tengo algún tipo de agencia, ésta se habilita por el hecho de que soy constituida por un mundo social que nunca elegí”, y "la posibilidad de mi persistencia como un 'yo' depende de mi posibilidad de hacer algo con lo que se hace de mí” (Butler, 2004, p. 3).

En segundo lugar, nos interesa agregar que para que ese trabajo transformador de desplazamiento e improvisación sea efectivo debe darse a través de un esfuerzo colectivo. Esto es porque, como nota Butler, "la relación crítica [con las normas] depende también de una capacidad, invariablemente 
colectiva, de articular una versión alternativa y minoritaria de normas básicas o de ideales que me permitan actuar" (Butler, 2004, p. 3). Las otras personas son quienes me reconocen, me nombran como quiero ser nombrado, y me recuerdan que esa existencia a la que quiero llegar es posible. En este punto es inevitable la referencia al film, en el que la obra de teatro sirve, entre otras cosas, como unión entre personas, construcción de un colectivo, y medio para llegar a una grupalidad. Los reclusos/actores se acompañan, reflexionan juntos sobre su propia realidad (inspirados en la trama de la obra), se dan apoyo e incluso se dicen cosas que nunca pudieron decirse. Por ejemplo, en una de las tantas escenas en las que cuesta reconocer cuándo termina el ensayo y empieza la vida en la prisión, dos internos discuten y el ambiente se llena de tensión; finalmente, algunos compañeros logran que vuelva la tranquilidad, y el ensayo continúa.

Este no es el único motivo por el que el ejercicio teatral nos sirve de ejemplo paradigmático de la transformación performativa. Si elegimos este film, es porque consideramos que las representaciones artísticas (en este caso, cine y teatro) pueden ser un medio privilegiado para ese retramado de la vida. Veamos algunos puntos que respaldan esta consideración.

Dijimos que las acciones humanas intencionales son acciones bajo descripciones, y que en el caso de una situación de privación de la libertad, las descripciones disponibles son muy limitadas. En proyectos como el narrado en César debe morir, el arte permite ampliar estas descripciones disponibles, no sólo porque se está emprendiendo un trabajo conjunto, y porque efectivamente se están representando personajes, sino también, y en un sentido más relevante, porque el trabajo sobre la obra permite a los reclusos pensarse de otra manera, pensar para sí mismos otra identidad más allá de la de "preso". El film muestra cómo allí "proyecto" se transforma en "posibilidad" -y en agencia. Vemos que desde los primeros momentos del ensayo los reclusos toman un lugar activo de decisiones sobre su propio desempeño: proponen modos de llevar adelante sus personajes, incorporan sus dialectos regionales, y toman como propio el desafío de transmitir el 
mensaje de la obra al público. En este sentido, los directores cuentan que los internos

venían de una vida donde el horizonte que tenían encima era un horizonte oscuro, lúgubre, en el que realmente no tenían valores... Entrando, en cambio, a la cárcel, poco a poco para muchos de ellos, o para algunos de ellos, mediante la cercanía con los grandes artistas, con los poetas que han escrito sobre el dolor humano, la esperanza humana... Poco a poco, recitando, su horizonte se abrió, entendieron realmente qué podía ofrecerles la vida (Suraci, 2013). ${ }^{8}$

En segundo lugar, hablamos de la necesidad de tener un espacio para "articular una versión alternativa" de normas e ideales. Butler misma reconoce que "la capacidad de desarrollar una relación crítica con estas normas presupone una distancia respecto de ellas, una habilidad de suspender o posponer la necesidad de ellas, aun existiendo un deseo de contar con normas que nos permitan vivir" (Butler, 2004, p. 3). Consideramos que la experiencia teatral de los reclusos habría sido clave al ofrecer este efecto "suspensor". Ella les permite mirar con cierta distancia (aunque, sabemos, no absoluta) su cotidianidad y su propia existencia para pensarlas de otra manera. De hecho, aquí la distancia se duplica al contar con un primer desdoblamiento entre la vida cotidiana y la obra de teatro, y un segundo desdoblamiento entre éstos y su reproducción cinematográfica. Sería posible seguir multiplicando estos desdoblamientos, si tenemos en cuenta también las proyecciones del film (en salas comerciales, festivales, etc.), y la vida de quienes, tras salir, continuaron una carrera como actores, tal como se relata en los títulos finales.

Por todo esto, podemos ver cómo en este caso es el arte, en cierto sentido, el que viene a responder al llamado inicial: “iHuye lejos, huye!”. Sin embargo, esa huida no toma la forma de una evasión, ni se ilusiona con una reconfiguración total. Más bien se da, en el caso del teatro, como un modo de

\footnotetext{
${ }^{8}$ Sin embargo, es importante notar (aunque no podamos detenernos en ella) la paradoja de este descubrimiento, resumida en aquella frase final de Casio citada más arriba. En palabras de los directores, "entonces, por un lado, ellos descubrieron el mundo en su variedad, en sus valores; por el otro, en el momento en que lo descubrieron, se dieron cuenta de que lo perdieron. Ahora, esto, es realmente una tragedia" (Suraci, 2013).
} 
retramar sus vidas (en términos del personaje que les tocara interpretar, y en términos de pensarse como "actores" con una tarea por cumplir). En tanto film, la huida toma la forma de una salida al "afuera", ya que el cine ofrece el medio para hacer pública esa nueva trama, ese cambio, a través de su reproductibilidad técnica.

\section{Algunas conclusiones}

A lo largo de estas páginas, las ideas de "matriz" y "performatividad" sirvieron como dos grandes ejes de análisis teórico para arrojar luz sobre aquello que, desde nuestro punto de vista, retrata César debe morir. En estrecha relación con el desarrollo de aquellos conceptos, pudimos marcar algunos puntos nodales del proceso de transformación personal y colectiva que muestra el film. Recordemos que éste cierra con la repetición de la escena inicial (un recurso que puede provocar cierta claustrofobia: hay algo que no cambia, cierta matriz institucional inevitable), pero le añade aquella confesión tan sugerente de Casio directamente a cámara (y con ella nos transmite: también hay algo profundo, personal y colectivo que ya no será igual). Por todo lo expuesto en nuestro trabajo, podemos decir que César debe morir apuesta en todo momento por la performatividad agenciadora en lugar de la aceptación pasiva de una matriz aplastante.

La película logra mantener distancia de las diversas formas de estigmatización que podrían devenir de la clasificación de sus protagonistas como "presos". Esta particularidad se manifiesta de diversas maneras a lo largo del film. Al momento de presentar a los internos/actores en el contexto del casting de la obra, los directores optan por que ellos mismos expresen su nombre, apellido, y lugar de nacimiento, sin requerir ninguna referencia a su delito y condena. Pero el film tampoco es ingenuo con respecto al peso de la situación de encierro, y nos muestra que quienes ven y sienten el estigma son los propios presos. Un ejemplo impactante de esto se encuentra en la escena en la que Brutto dice una frase de su parlamento que le recuerda su propia biografía: “ojalá pudiera arrebatar el espíritu del tirano sin abrirle el pecho”. 
El hombre queda abatido por el recuerdo que disparó esa frase, y piensa cómo hubiera seguido la historia si en aquel momento él hubiera pensado como Brutto ahora. El deseo de seguir con el ensayo, de seguir siendo Brutto, y no ese hombre del pasado, lo hace levantarse y continuar. De hecho, "Brutto terminó su condena y se convirtió en actor en vez de perder la batalla contra Octavio y suicidarse” (Pujato, 2013, p. 35).

Por otro lado, si bien la película muestra los verdaderos nombres de los reclusos en el momento del casting, a partir de la asignación de los roles principales y hasta el final se los llama por su nombre ficcional. En el contexto de la obra y de la película no importa su pasado: ellos no son quienes llegaron a la cárcel, sino las personas/personajes que están construyendo ahora. Leemos esto como otra apuesta al potencial transformador, "el increíble poder y la grandeza del arte” que retrata el film (Mattanza, 2012).9 Con el trabajo en la obra, los internos tienen la posibilidad de pensarse de otra manera, pensar para sí mismos otra identidad más allá de la de "preso". Esto habilita a una identidad activa, que los aleja del lugar pasivo del sufrimiento o la victimización.

Julio César es, sin dudas, una obra trágica - y hay mucho de trágico en la vida de estas personas. Pero la puesta en escena de esta tragedia en el film hace de ella un medio por el cual sus protagonistas pueden hacer de esa experiencia artística bifronte un elemento nuevo, una herramienta para modificar su propia trama, para empezar a vivir su vida de otra manera. Por todo esto, creemos que la película, finalmente, nos dice: César debe morir, para que algo nuevo pueda nacer.

\section{Referencias bibliográficas}

Austin, J. L. (1975). How To Do Things With Words. Segunda edición. Cambridge: Harvard University Press.

Butler, J. (2002). Cuerpos que Importan. Sobre los límites materiales y discursivos del 'sexo'. Buenos Aires: Paidós. [1993]

Butler, J. (2004). Undoing Gender. Nueva York: Routledge.

9 Afirman los directores: "para nosotros, el film cuenta principalmente el descubrimiento del increíble poder y de la grandeza del arte" (Mattanza, 2012). 
Goffman, E. (2001). Internados. Buenos Aires: Amorrortu. [1961]

Goffman, E. (2006). Estigma. La identidad deteriorada. Buenos Aires: Amorrortu. [1963]

Hacking, I. (1999). The social construction of what? Cambridge: Harvard University Press.

Hacking, I. (2000). Historical Ontology. Cambridge: Harvard University Press.

Mattanza, A. (2012). "I fratelli Taviani verso l'Oscar". Oggi, 26 de septiembre de 2012. Disponible en: http://www.oggi.it/people/programmi-tvspettacoli/2012/09/26/i-fratelli-taviani-verso-loscar/

Pujato, F. (2013). "Fuera de prisión (el cine)". Cinéfilo, nº 4 (14), pp. 34-35.

Suraci, D. (2013). "Entrevista a Paolo y Vittorio Taviani sobre su film César debe morir”. Instituto Italiano de Cultura de Bogotá. Disponible: https://www.youtube.com/watch?v=ncSAjXXoQik

Taviani, P. y Taviani, V. (2012). Cesare deve morire. Italia: Kaos Cinematografica, Rai Cinema, Stemal Entertainment, Le Talee.

Cómo citar: Pérez, M. y Bevilacqua, G. (2016). "Identidades estigmatizadas y su refiguración performativa en César debe morir". Fotocinema. Revista científica de cine $y$ fotografía, 12, pp. 263-283. Disponible: http://www.revistafotocinema.com/ 